\title{
Triaging, emergency surgical management, and nutritional rehabilitation of a case of acute on chronic mesenteric vascular ischemia
}

\author{
Jeena Raju Kudunthail ${ }^{1}$, Asif Ansari ${ }^{2}$, Waqar Ansari ${ }^{3}$, Kushagra Rahul Srivastav ${ }^{4}$ \\ From ${ }^{1}$ Senior Resident, ${ }^{2}$ Honorary Assistant Professor, ${ }^{3}$ Honorary Head of Unit, ${ }^{4}$ Senior Resident, Department of General Surgery, GGMC, Mumbai, \\ Maharashtra, India
}

\begin{abstract}
Mesenteric vascular ischemia (MVI) is a leading cause of short bowel syndrome (SBS). There is no uniformity in management protocol for MVI and there lacks consensus on when and how to operate. In this report, we present a case of type 2 SBS following extensive resection of bowel managed postoperatively with a combination of enteral nutrition (EN) and parenteral nutrition (PN). We used Acute Physiology and Chronic Health Evaluation 2 scoring system along with intraoperative findings to stratify our case and proceed with resection and anastomosis of the bowel in the same sitting. The total PN was started from post-operative day 1 along with micronutrients and vitamin supplements. We were able to taper PN to supplement $30 \%$ of the daily caloric requirement and $70 \%$ by EN by the end of 8 weeks. Triaging the patient, prompt surgery, early institution of PN along with EN, micronutrients, and vitamin supplements proved to be the key for improved outcomes.
\end{abstract}

Key words: Acute physiology and chronic health evaluation 2 score, Parenteral nutrition, Short bowel syndrome, Thrombosis

$\mathrm{M}$ esenteric vascular ischemia (MVI) results when the perfusion of the abdominal viscera fails to meet the normal metabolic requirements. It can either be acute or chronic [1]. Acute mesenteric ischemia is most commonly due to acute thrombosis related to a pre-existing plaque or embolization from a distant site [2,3]. Chronic mesenteric ischemia has a more insidious process and progresses over weeks to several months. Most patients with chronic mesenteric occlusive disease manifest no symptoms, hence, the exact incidence is not known. The patients usually demonstrate the involvement of $\geq 2$ mesenteric vessels before symptoms arise, resulting from the development of significant collateral vessels overtime [4].

There is an increasing incidence of mesenteric ischemia yet there is no enlisted protocol for how to manage such cases. In this report, we describe a case of acute on chronic mesenteric ischemia involving the superior mesenteric artery (SMA), inferior mesenteric artery (IMA), and superior mesenteric vein (SMV) in a 60-year-old hypertensive male using the Acute Physiology and Chronic Health Evaluation 2 (APACHE-2) score.

\section{CASE REPORT}

A 60-year-old gentleman presented to the casualty with complaints of sudden onset abdominal pain and distension with vomiting for

\section{Access this article online}

Received - 02 May 2021

Initial Review - 18 May 2021

Accepted - 22 May 2021

DOI: $10.32677 /$ IJCR.2021.v07.i06.001
2 days. He had a 2-day history of obstipation. On further inquiry, the patient narrated a history of indigestion and dull aching postprandial abdominal pain for over 1 month.

On examination, he was anxious with a pulse rate of $110 / \mathrm{min}$, blood pressure of 130/80 $\mathrm{mmHg}$, and respiratory rate of $22 / \mathrm{min}$ with an oxygen saturation of $98 \%$ on room air. The abdomen was visibly distended with generalized tenderness and no bowel sounds on auscultation. On per rectal examination, the rectum was collapsed with no stool staining. A nasogastric tube was inserted with $400 \mathrm{cc}$ of bilious return.

Contrast-enhanced computed tomography scan of abdomen and pelvis revealed thrombosis of SMA, IMA, and SMV along with a lack of contrast enhancement of bowel with pneumatosis intestinalis (Fig. 1). This confirmed the diagnosis of mesenteric ischemia with bowel necrosis. The patient's APACHE-2 score was calculated and found to be 9 [5]. The patient was hemodynamically optimized and planned for emergency laparotomy.

A midline vertical incision was employed. The small bowel loops were necrotic from $30 \mathrm{~cm}$ distal to the duodenojejunal flexure till the hepatic flexure of the transverse colon. There was no peritoneal soiling or perforation of the viscus. Within the bowel mesentery, SMA pulsations were absent, while those of the IMA were feeble. The necrotic bowel was resected and jejunal transverse anastomosis was performed (Fig. 2). A thorough intraperitoneal lavage with normal saline was given and two abdominal drains were kept, one in Morrison's space and the

Correspondence to: Jeena Raju Kudunthail, Room Number 15, Building Number 2, Old BMC Building, Hemant Manjrekar Road, Raoli Camp, Mumbai, Maharashtra, India. E-mail: drjeenakr@gmail.com

(C) 2021 Creative Commons Attribution-NonCommercial 4.0 International License (CC BY-NC-ND 4.0). 


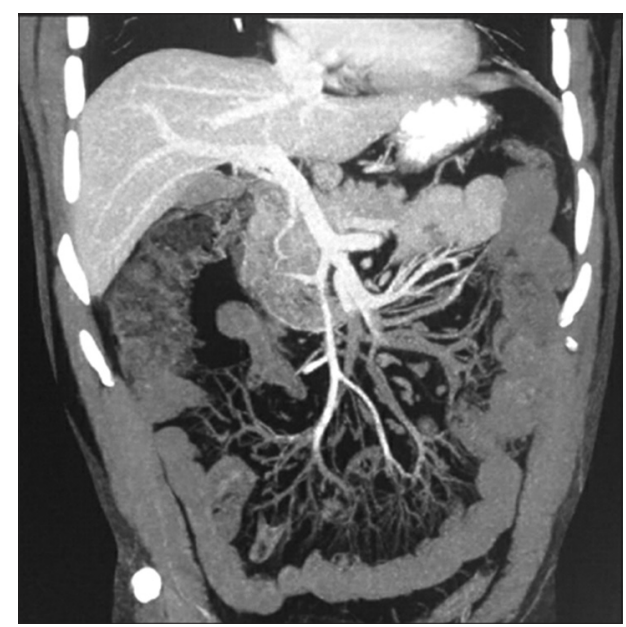

Figure 1: Computed tomography showing superior mesenteric artery and inferior mesenteric artery block

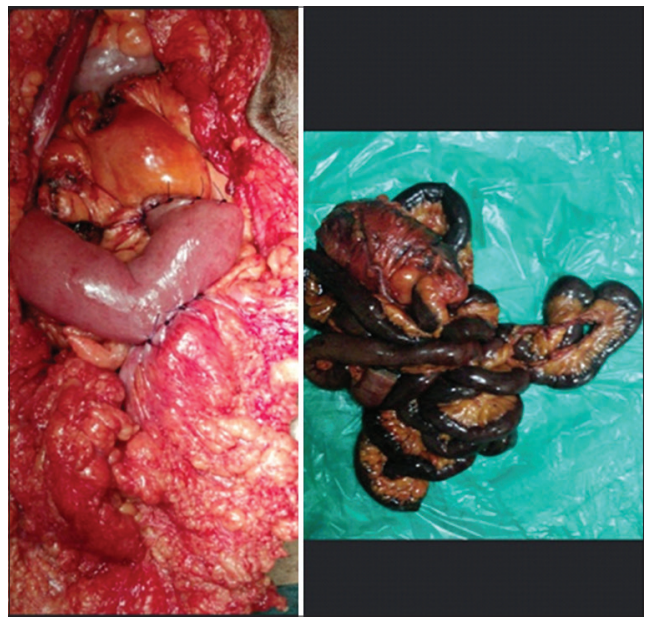

Figure 2: Left image showing jejunotransverse side-to-side anastomosis and the right image showing resected bowel

other in the pelvis. The total operative time was $170 \mathrm{~min}$, with an estimated blood loss of $200 \mathrm{~mL}$.

The patient was kept in the intensive care unit for 2 days without the need for any ventilatory support. We commenced anti-thrombotic therapy in the form of intravenous unfractionated heparin $12 \mathrm{~h}$ following surgery. Total PN was commenced on the $1^{\text {st }}$ post-operative day after calculating the total daily caloric requirement using the Harris-Benedict equation which came up to $1659 \mathrm{kcal}$ [5]. About $30 \%$ of this requirement was given parenterally on the $1^{\text {st }}$ post-operative day, $50 \%$ on the $2^{\text {nd }}$ day, and $70 \%$ from the $3^{\text {rd }}$ day onward. On a post-operative day 3 , the patient was started on warfarin $3 \mathrm{mg}$, aspirin $75 \mathrm{mg} \mathrm{OD}$, and atorvastatin $40 \mathrm{mg}$ HS. The oral liquid diet was given from post-operative day 5 , subsequently shifted to a home-based soft diet followed by a full diet. The total fluid intake was at least $2.5-3 \mathrm{~L} /$ day, with not more than $200 \mathrm{ml}$ of liquid per intake. Parenteral Vitamin B12 supplementation was given along with oral supplements of loperamide, ferrous sulfate with folic acid, Vitamin A, Vitamin E, calcium with Vitamin D3, and glutamine [6]. Stool consistency and frequency changed from liquid 6-8 times/day to semisolid 3-4 times/day. Daily monitoring of electrolytes and daily weight

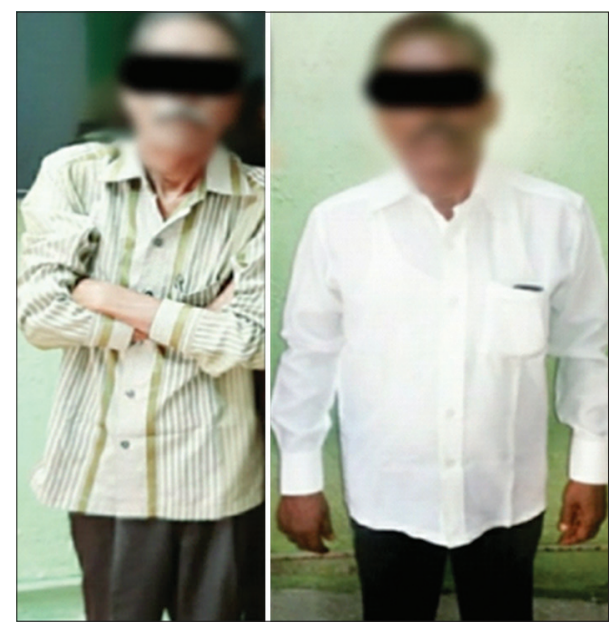

Figure 3: Left image is showing the patient at the time of discharge and the right image is showing the patient at 12-month follow-up

charting were done for 2 weeks. The patient was discharged from the hospital on a full diet after 8 weeks (Fig. 3).

\section{DISCUSSION}

Acute mesenteric ischemia accounts for only $0.09-0.2 \%$ of all admissions to the emergency department. Prompt diagnosis and intervention are required to reduce the high mortality rate of $50-80 \%[7]$.

In case of extensive necrosis of most of the small bowel with or without a portion of the colon, the surgeon could face a philosophical decision whether to do anything at all. Resection of the entire involved bowel will result in short bowel syndrome (SBS) with its associated complications. This may not be preferable in elderly infirm patients who may not tolerate longterm PN. A pre-operative discussion with the patient and the patient's family concerning these issues should be undertaken to decide further management $[7,8]$.

Sun et al. used the APACHE-2 scoring system to predict the occurrence of irreversible intestinal ischemia with the early identification of patients who ultimately require surgical resection [9]. Mani et al. used the APACHE-2 scoring system to triage, assess the severity, and surgically manage patients with intra-abdominal sepsis following small bowel perforations of any etiology [5]. We have used the APACHE-2 scoring system to help categorize the patient and to decide whether primary anastomosis can be performed in this particular case. The APACHE- 2 score if $<10$, primary anastomosis can be planned, and if $\geq 10$, the patient needs damage control surgery. The score was 9 and the patient was planned for emergency laparotomy [5].

In this case, the patient had thrombosis of SMA, IMA, and SMV along with extensive bowel necrosis. Due to the extensive involvement of the vessels and as necrosis of the bowel had already set in, a revascularization procedure was not performed and medical anticoagulation was started postoperatively at the earliest. APACHE- 2 score $<10$ with no intra-abdominal contamination prompted for a single-step procedure with the restoration of bowel continuity (Type 2 SBS) [10]. The patient 
was given a combination of EN and supplementary PN along with a double strength dosage of oral fat-soluble vitamins and injection Vitamin B12 supplements. The patient is on follow-up for the past 12 months and has started gaining weight with an overall sense of well-being.

\section{CONCLUSION}

MVI has a high rate of morbidity as well as mortality. Most of the patients with extensive resection require lifelong supplemental nutrition which is not without its own complications. Early categorization of patients requiring prompt surgical management, appropriate early institution of PN along with EN, customized diet plan, and judicious follow-up are instrumental in not only prolonging survival but also improving the quality of life. This report focuses on the importance of formulating a protocol for managing such cases.

\section{REFERENCES}

1. Schermerhorn ML, Giles KA, Hamdan AD, Wyers MC, Pomposelli FB. Mesenteric revascularization: Management and outcomes in the United States, 1988-2006. J Vasc Surg 2009;50:341-8.e1.

2. Lock G. Acute intestinal ischaemia. Best Pract Res Clin Gastroenterol 2001;15:83-98.
3. Stroh CI, Shemesh J, Motro M. Management of acute mesenteric ischemia. N Engl J Med 1996;335:328-31.

4. Thomas JH, Blake K, Pierce GE, Hermreck AS, Seigel E, Gerwertz BL. The clinical course of asymptomatic mesenteric arterial stenosis. J Vasc Surg 1998;27:840-4.

5. Mani C, Borah C, Krishna SV, Anuj M, Bhatnagar D. The role of APACHE-II triaging in optimum management of small bowel perforations. Trop Doct 2001;31:198-201.

6. Wall EA. An overview of short bowel syndrome management: Adherence, adaptation, and practical recommendations. JAcad Nutr Diet 2013;113:1200-8.

7. Bala M, Kashuk J, Moore EE, Kluger Y, Biffl W, Gomes CA, et al. Acute mesenteric ischemia: Guidelines of the world society of emergency surgery. World J Emerg Surg 2017;12:1-11.

8. Lilley EJ, Cooper Z, Schwarze ML, Mosenthal AC. Palliative care in surgery: Defining the research priorities. J Palliat Med 2017;20:702-9.

9. Sun SL, Wang XY, Chu CN, Liu BC, Li QR, Ding WW. Predictors of irreversible intestinal resection in patients with acute mesenteric venous thrombosis. World J Gastroenterol 2020;26:3625-37.

10. Keller J, Panter H, Layer P. Management of the short bowel syndrome after extensive small bowel resection. Best Pract Res Clin Gastroenterol 2004; 18:977-92.

Funding: None; Conflicts of Interest: None Stated.

How to cite this article: Kudunthail JR, Ansari A, Ansari W, Srivastav KR. Triaging, emergency surgical management, and nutritional rehabilitation of a case of acute on chronic mesenteric vascular ischemia. Indian J Case Reports. 2021;7(6):223-225. 